\title{
Somatotipo de mujeres futbolistas universitarias por posición en el terreno de juego Somatotype of university female soccer players by playing position on the field
}

\author{
Alejandra Caballero-Ruíz, Claudia E. Carrasco-Legleu, Lidia G. De León, Ramón Candia-Luján, Briseidy Ortiz-Rodríguez
} Universidad Autónoma de Chihuahua (México)

\begin{abstract}
Resumen: El objetivo del presente trabajo fue caracterizar el somatotipo en una muestra de futbolistas universitarias de una institución del norte de México, de acuerdo a su posición en el terreno de juego y compararlo contra el somatotipo de un equipo homólogo latinoamericano. Participaron 19 jugadoras de futbol soccer pertenecientes al equipo representativo de una Universidad del norte de México en la temporada competitiva. Las jugadoras fueron clasificadas por su posición en el campo, en tres categorías: defensas, medias y delanteras. Se determinó el somatotipo a partir de 10 variables antropométricas de acuerdo al método Heath \& Carter y se comparó con un referente de jugadoras universitarias campeonas en su país pertenecientes a la Pontificia Universidad de Valparaíso (PUCV). La categoría general de las jugadoras universitarias fue endomorfo - mesomorfico con un somatotipo 4.3-3.6-2.0, y con una distancia somatotipica (SAD) entre grupos mayor a una unidad del referente homologo. Las jugadoras evaluadas presentaron un somatotipo similar por posición de juego con un SAD menor a 1, las defensas y medias fueron endomorfo-mesomorfico, mientras que las delanteras endomorfo-mesomorfo. En el grupo evaluado no existieron diferencias significativas por posición de juego, por lo que se hubiera esperado diferencias por las exigencias dentro del terreno de juego. El componente de adiposidad relativa resultó dominante en la muestra evaluada respecto al referente utilizado en el presente estudio, lo que podría ser un factor que influya en el rendimiento deportivo.
\end{abstract}

Palabras Clave: antropometría, somatotipo, futbol soccer.

Abstract: The aim of the present study was to characterize the somatotype of a sample of university female soccer players from an institution in the north of Mexico according to their position on the field, and to compare them against the somatotype of a similar team from Latin America. A total of 19 soccer players from the university representative team participated in the study, developed during the competitive season. The players were classified in three categories based on their position on the field: defenses, midfielders, and forwards. Anthropometric somatotypes were determined using 10 variables according to the Heath \& Carter method, and they were compared with those of athletes from the Pontifical University of Valparaíso (PUCV), who are champions in their country. The overall category of the players evaluated was mesomorphic endomorph with a 4.3-3.6-2.0 somatotype, with a difference bigger than 1 unit in the somatotype distance (SAD) with the PUCV reference team. The evaluated players have a similar somatotype according to their position, where SAD does not show bigger difference than 1 . Defenses and midfielders presented mesomorphic-endomorph, whereas forwards mesomorph-endomorph somatotype. In the evaluated group, there were no significant differences by position on the field, which is unexpected considering the different requirements of the game per position. The relative adiposity component proved to be dominant in the players from our sample compared to the reference team, which could be a factor that influences sport performance.

Keywords: Anthropometry, somatotype, soccer.

\section{Introducción}

Hoy en día un mayor número de mujeres participan en actividades deportivas, por lo que la evolución de la mujer en el deporte ha captado la atención de investigadores a nivel mundial, ya que han logrado alcanzar excelente forma atlética en diversas disciplinas.

El fútbol es uno de los deportes más populares en la actualidad. La Federación Internacional de Fútbol Asociación (FIFA), menciona que existen más de 30 mil jugadoras federadas y no federadas en todo el mundo, la mayoría en Estados Unidos y Canadá. También, se ha documentado que hay un mayor número de mujeres futbolistas en Europa y Oceanía que en Asia, Sudamérica y África. En México también se ha observado un aumentado de mujeres que juegan fútbol, en el año 2000 se tenían registradas más de doce mil jugadoras y para el 2006 más de quince mil (FIFA 2006), dicho incremento de mujeres en esta disciplina hace necesario más investigación sobre la composición corporal de las atletas de acuerdo a cada disciplina y establecer si es determinante para lograr un mejor rendimiento deportivo (Bahamondes et al., 2012).

El conocimiento y análisis de la forma corporal es un

Fecha recepción: 12-03-18. Fecha de aceptación: 05-02-19 Briseidy Ortiz Rodríguez bortizr@uach.mx elemento importante en el deporte, ya que es indudable que determinadas características físicas estén ligadas al máximo desempeño deportivo, dando fundamento al prototipo morfológico que puede aportar valiosa información respecto a dimensiones corporales de jugadores élite (Reilly et al., 2000). La determinación de las características físicas puede ser de utilidad de manera individual y en consecuencia para el equipo, con el fin de mejorar métodos de entrenamiento, alimentación, ubicación de las jugadoras dentro del terreno de juego y la selección de atletas (Zuñiga et al., 2018; Fernández et al., 2017; Fidelix et al. 2014; Rodríguez \& Echegoyen 2005).

Algunos estudios sugieren que existen diferencias entre el fútbol femenino y masculino, esto podría ser debido a estrategias de juego, condición física y morfología desiguales, además de presentar una detección tardía de la habilidad y un menor tiempo de entrenamiento de acuerdo al nivel competitivo. Específicamente en mujeres, es importante generar programas de entrenamiento considerando las características de maduración por grupos de edad, para comprender las demandas por este deporte, lo que hace necesario identificar dichas características para encontrar un perfil óptimo (Barbero-Álvarez et al., 2008).

Debido a los pocos referentes en el ámbito femenino en cuanto a las características morfológicas que sean la base de un mejor desempeño físico en el campo, el objetivo del presente estudio fue caracterizar el somatotipo antropométrico 
en una muestra de futbolistas universitarias del norte de México, de acuerdo a su posición en el terreno de juego y compararlo contra el somatotipo de un equipo homólogo latinoamericano.

\section{Material y método}

Participantes. Se reclutaron 19 jugadoras de fútbol soccer pertenecientes al equipo representativo de una Universidad del norte de México, de entre 17 y 25 años de edad. Cada deportista firmó una carta de consentimiento informado de participación voluntaria, en el cual se le explicó detalladamente el procedimiento del estudio. Los procedimientos se realizaron bajo los principios establecidos en la declaración de Helsinki (Asociación Médica Mundial 2014). La recolección de los datos se efectuó en el periodo competitivo del macrociclo de entrenamiento.

Instrumentos. Para la determinación del Somatotipo antropométrico se utilizó un Tom Kit de Rosscraft. Una báscula digital modelo 1631 solar scale y un estadímetro modelo 202, Seca Ltd, para peso corporal y estatura máxima, respectivamente. Para la medición de pliegues cutaneos se utilizó un plicómetro Slimguide ${ }^{\circledR}$, una cinta antropométrica Lufkin para circunferencias y un vernier Tommy 3 para diámetros cortos.

Procedimiento. Las jugadoras fueron clasificadas en tres grupos según su posición en el campo: defensas (D), medias (M) y delanteras (DL); las porteras no se incluyeron en el estudio debido a que solo era una. Todas las mediciones antropométricas se realizaron por duplicado bajo los lineamientos de la Sociedad Internacional para el Desarrollo de la Cineantropometría, por sus siglas en inglés (ISAK) y realizadas por dos antropometristas certificados en el nivel III de ISAK(Stewart et al., 2011). Se realizó la medición de las 10 variables incluidas para determinar somatotipo de acuer-

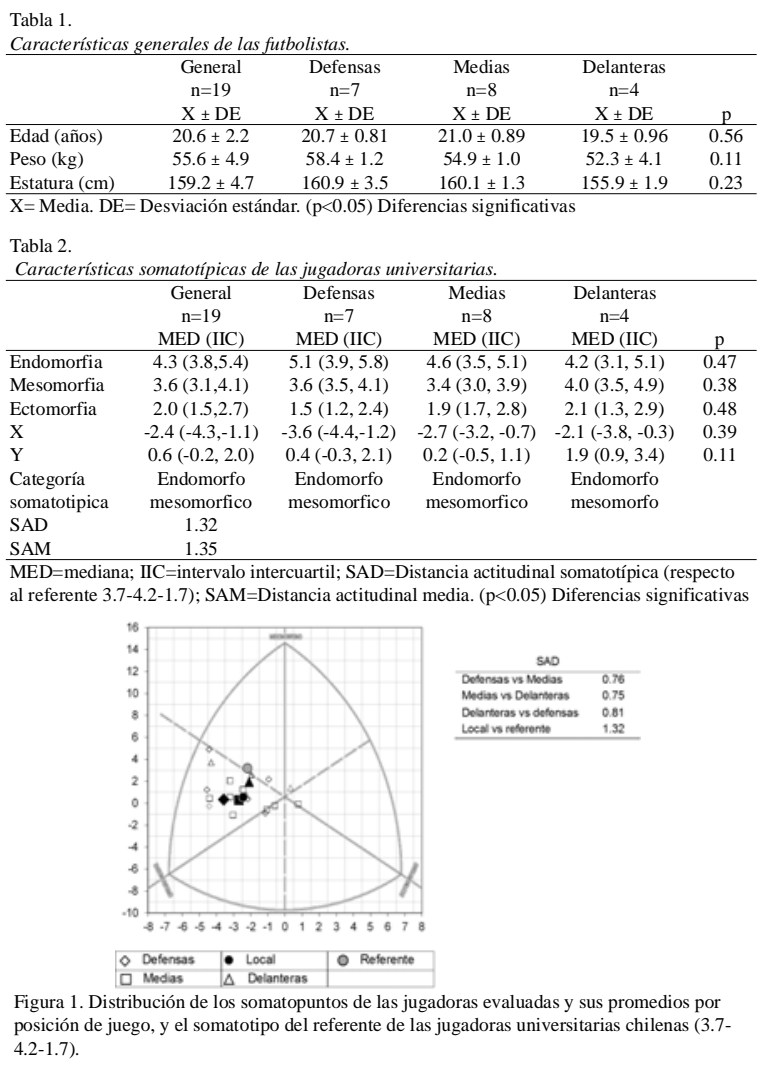

do al método Heath \& Carter: dos medidas básicas, peso corporal y estatura máxima; cuatro panículos adiposos (tríceps, subescapular, supraespinal y pierna medial), dos circunferencias (brazo tensionado y pierna máxima) y dos diámetros (húmero y fémur). Para el calculo del somatotipo se emplearon las ecuaciones descritas por Heath \& Carter (1990). Se determino la distancia posicional tridimensional somatotípica (Somatotype Atitudinal Distance) y la distancia posicional media (Somatotype Atitudinal Media), SAD y SAM respectivamente por sus siglas en inglés, según el procedimiento descrito por Heath \& Carter (1990). Las ecuaciones para obtener el SAM y el SAD son como sigue: SAM = Sumatoria de SAD/n, SAD A, B = Raíz [(endomorfiaA - endomorfia $\mathrm{B})^{2}+(\text { mesomorfia } \mathrm{A} \text {-mesomorfia } \mathrm{B})^{2}+$ (ectomorfiaA + ectomorfia B ${ }^{2}$ ], donde A y B son sujeto 1 y sujeto 2, respectivamente.

Para el análisis estadístico se utilizó el programa SPSS ${ }^{\circledR}$ (versión 18.0 IBM para Windows); los datos se encontraron no normales después de la prueba de Shapiro Wilk. Se utilizó Kruskal - Wallis para muestras independientes y los resultados se presentan en medianas y rango intercuartil entre el 25 y 75 percentil (IIC).

\section{Resultados}

En la tabla I se muestran los valores de edad, peso y estatura por su posición ocupada en el terreno de juego. No se observan diferencias significativas en las variables antes mencionadas.

En la tabla II se presentan valores de endomorfia, mesomorfia y ectomorfia, además de la categoría del somatotipo, SAM y SAD en función de la posición de juego en el campo. Sin encontrar diferencias significativas.

En la figura 1 se presenta la somatocarta por posición de juego donde se observa una dispersión entre las jugadoras evaluadas con un predominio del componente endomorfo, las defensas y medias se encuentran en una categoría somatotipica endomorfo - mesomorfico y las delanteras endomorfo - mesomorfo. Respecto al SAD por posición de juego no difiere de 1 por lo que el grupo es homogéneo.

\section{Discusión}

El hecho de que el somatotipo en general fuera distinto al del referente homólogo, nos permitió establecer que no existe homogeneidad entre los grupos, aún y cuando practican la misma disciplina deportiva. Es probable que el predomino del somatotipo endomorfo-meosomorfico en la muestra evaluada, influya en el rendimiento a nivel competitivo.

Se ha reportado que la estatura no es un determinante para el rendimiento deportivo, ya que en el mundial 2011 se reportaron jugadoras desde $152 \mathrm{~cm}$ hasta $187 \mathrm{~cm}$, siendo Japón las que registraron menor estatura pero eran más dinámicas, rápidas y con un juego de balón al ras del suelo lo que les permitía mantener un mayor control sobre el balón (FIFA 2011). Sin embargo, las defensas requieren ser altas para cortar el juego aéreo, así como para jugadas tácticas ofensivas.

A diferencia de lo encontrado en el presente estudio, otros reportes de jugadoras chilenas profesionales, universitarias y en jugadoras españolas de primera división regio- 
nal y nacional presentaron un somatotipo endomorfomesomorfo (Almagiá et al., 2008; Barraza et al., 2015; Sedano et al., 2009). Por lo que al resultar las deportistas chilenas universitarias campeonas (referente homólogo), se pudiera confirmar que el hecho de presentar un equilibrio entre los componentes de su somatotipo, parecen jugar un papel determinante para un mejor desempeño dentro del terreno de juego.

Al observar el valor de SAD (>1), confirma que el somatotipo es diferente entre las jugadoras locales versus el referente homólogo, lo que podría indicar que las diferencias en la distribución del somatotipo, son observadas en futbolista de alto rendimiento. Con lo anterior, se podría sugerir que el predominio balanceado de endomorfia y mesomorfia (grasa y musculo) puede ser el modelo a seguir para un somatotipo ideal en jugadoras de futbol soccer femenil, ya que se manifiesta a nivel profesional y en universitarias campeonas. Sin embargo, Adhikari \& Nugent (2014) propone que de acuerdo a las demandas físicas dentro del terreno de juego se esperaría que las jugadoras en general presentaran una categoría somatotípica mesomorfo-ectomorfica, ya que menciona que para el fútbol, se requiere velocidad y resistencia con potencia muscular, con valores bajos en el componente de endomorfia se mejorarían estas capacidades físicas.

En un estudio realizado por Barraza et al., (2015) se encontró que en las posiciones defensivas existió un predominio de la endomorfia al compararlas con las jugadoras de ataque, en la muestra evaluada se identificó que dicho componente predominio en defensas y medias, no siendo así en las delanteras; sin embargo, se hubiera esperado encontrar diferencias en cuanto a las características del somatotipo por posición de juego debido ya que las demandas físicas son distintas en cada posición en el terreno de juego.

Underwood (2009) menciona que en los delanteros predominan acciones de velocidad de reacción y velocidad máxima frente a recorridos de alta duración. Además, el sistema de juego podría ser fundamental en las demandas físicas de las jugadoras aunado a un somatotipo específico, aun cuando Bradley et al., (2011), no encontró diferencias significativas sobre las demandas físicas de los jugadores de manera individual, al realizar un estudio entre sistemas de juego, menciona que en el sistema 4- 3- 3, los delanteros realizaban un $30 \%$ más de carrera de alta intensidad con y sin posesión de la pelota, que en 4-4-2 y 4-5-1. En este sentido, las jugadoras locales tienen un predominio en el sistema 4-42 en el que las demandas de juego no son diferentes de acuerdo a Bradley, por lo que pudiera ser que no se encontraron diferencias significativas entre las jugadoras.

Sería conveniente realizar estudios con un mayor número de jugadoras y a su vez dividir en al menos seis categorías, los cuales permitirían dar a conocer características específicas y con mayor idoneidad para las posiciones dentro del terreno de juego (Martínez-Lagunas et al., 2014).

Una vez caracterizado el somatotipo de las jugadoras por posición de juego tenemos las bases necesarias para trabajar enfocados a un mayor rendimiento dentro del terreno de juego, a través de un equipo de trabajo multidisciplinar (director técnico, preparador físico, nutriólogo, científico del deporte, psicólogo, médico del deporte), necesarios en los equipos representativos quienes trabajan a la par con las atletas para potencializar las capacidades físicas, técnicas y tácticas; personalizando el entrenamiento por posición de juego; esto acompañado de una alimentación saludable, realizando evaluaciones antropométricas y de capacidades físicas periódicas a fin de ir mejorando su rendimiento en los distintos momentos del macrociclo (Rivera, 2006).

\section{Conclusiones}

A pesar de que existe una aparente homogeneidad en el somatotipo de las jugadoras evaluadas, es importante que cada posición de juego posea una distinta distribución del somatotipo en relación a la media del grupo y de acuerdo a la posición en el terreno de juego que permita una clara selección de talentos.

\section{Referencias}

Adhikari, A. \& Nugent, J. (2014) Anthropometric characteristic, body composition and Somatotype of Canadian female soccer players. American Journal of sports science, 2(6-1), 14-18.

Almagià, A. F., Rodríguez, R. F. J., Barraza, G. F. O., Lizana, P. J. \& Jorquera, A. C. A. (2008) Perfil antropométrico de jugadoras chilenas de futbol femenino. International Journal of Morphology, 26(4), 817-821.

Asociación Médica Mundial. (2014). Declaración de Helsinki. Principios éticos para la investigación en seres humanos. Boletín del Consejo Académico de Ética en Medicina. 1(2): 239-243.

Bahamondes, A. C., Cifuentes, C. B. M., Lara, P. E. \& Berral, R. F. J. (2012) Composición corporal y somatotipo en futbol femenino. Campeonato sudamericano sub17. International Journal of Morphology, 30(2), 450-460.

Barraza, F., Yáñez, R., Báez, E. \& Rosales, G. (2015) Características antropométricas por posición de juego en mujeres futbolistas chilenas de la Región de Valparaíso. International Journal of Morphology, 33(4), 1225-1230.

Barbero-Álvarez, J. C., Gómez, L. M., Barbero, A. V., Granda, J., Castanga, C. J. (2008). Heart rate and activity profile for young female soccer players. Journal of Human Sport Exercise, 3(2), 1-11.

Bradley, P. S., Carling, C., Archer, D., Roberts, J., Dodds, A., Di Mascio, M., Paul, D., Diaz, A. G., Peart, T., \& Krustrup, P. (2011) The effect of playing formation on high-intensity running and technical profiles in English Premier League soccer matches. Journal of Sports Science, 29, 821-830. https://doi.org/10.1080/ 02640414.2011.561868

Carter, J. E. L. \& Heath, B. H. (1990) Somatotyping - Development and applications. Cambridge studies in biological anthropology. Cambridge, University Press.

Fidelix, Y. L., Berria, J., Ferrari, E. P., Ortiz, J. G., Cetolin, T. \& Petroski, E. L. (2014) Somatotype of competitive youth soccer players from Brazil. Journal of Human Kinetics, 42, 259.

Federación Internacional de Futbol Asociación FIFA. Women's football today information and statistics on women's football from the members associations of FIFA. Versión online consultada en agosto del 2017. http:// resources.fifa.com/mm/document/afdeveloping/women/93/77/21/ factsheets.pdf

Fernández, A. J., Rubiano, P. A., \& Hoyos, L. A. (2017). Perfil morfológico de voleibolistas de altos logros. Revisión. Revista Internacional de Medicina y Ciencias de la Actividad Física y del Deporte, 17(68), 775-794.

Martinez-Lagunas, V., Niessen, M., \& Hartman, U. (2014) Women’s football: Player characteristics and demands of the game. Journal of Sport and Health Science, 3(4), 258-272.

Reilly, T., Bangsbo, J., \& Franks, A. (2000) Anthropometric and physiological predispositions for elite soccer. Journal of Sports Sciences, 18(9), 669-683. https://doi.org/10.1080/02640410050120050

Rivera-Sosa, J. M. (2006). Valoración del somatotipo y proporcionalidad de futbolistas universitarios mexicanos respecto a futbolistas profesionales. Revista Internacional de Medicina y Ciencias de la Actividad Física y del Deporte/International Journal of Medicine and Science of Physical Activity and Sport, 6(21), 16-28.

Rodríguez, G. C., \& Echegoyen, M. S. (2005) Características antropométricas fisiológicas de jugadores de futbol de la selección mexicana. Archivos de Medicina del Deporte, 22(105), 33-37.

Sedano, S., Cuadrado, G., Redondo, J.C. \& De Benito, A. (2009) Perfil antropométrico de las mujeres futbolistas españolas. Análisis en función del nivel competitivo y de la posición ocupada habitualmente en el terreno de juego. Apunts educación física y deporte, 98, 78-87.

Stewart, A; Marfell-Jones, M; Olds, T; \& De Ridder H. (2011) Protocolo internacional para la valoración antropométrica. United Kingdom: Sociedad Internacional para el Avance de la Cineantropometria.

Underwood, G. (2009) Preparación Física en la Edad Infanto - Juvenil. Cid Editorial/ apuntes, 1-23.

Zuñiga, G. U., Osorio, G. A., Toledo, D. I. D. J. \& Herrera, P. R. (2018). Somatotipo en futbolistas mexicanos profesionales de diferente nivel competitivo. Retos (34), 100-102 